\title{
Características y vulnerabilidades del sector exportador cubano en el periodo revolucionario (1959-2013)
}

\author{
Characteristics and Vulnerabilities \\ of Cuban Export Sector in \\ the Revolutionary Period (1959-2013)
}

\author{
Francisco García Fernández \\ Universidad Autónoma de Tamaulipas, Tamaulipas, México, \\ email: ffernandez@docentes.uat.edu.mx
}

Bruno Sovilla

Universidad Autónoma de Chiapas, Chiapas, México, email: brunosov@yahoo.it

\begin{abstract}
Resumen. Este trabajo analiza las transformaciones del sector exterior cubano a través de la evolución de un grupo de indicadores seleccionados en el periodo revolucionario entre 1959 y 2013 para identificar las principales vulnerabilidades que enfrenta en el corto y mediano plazos. Se siguió un enfoque histórico descriptivo basado en la evolución de variables económicas en el tiempo (1959-2013). Se observó que el sector exportador cubano ha sufrido cambios importantes, aunque ha mantenido debilidades estructurales, a lo largo del periodo considerado. A partir de 1989, Cuba tuvo que ajustar sus relaciones comerciales frente a la inesperada caída de los regímenes socialistas europeos con los cuales tenía relaciones privilegiadas. En la etapa sucesiva el comercio se ha diversificado, pero ha conservado la relación de dependencia con países ideológicamente afines, y con eso las debilidades mencionadas que en el corto plazo vislumbran un panorama poco alentador.
\end{abstract}

Palabras clave: sector exportador cubano; diversificación; tasa de apertura; economía cubana.

Abstract. This work is aimed at examining the transformations in the Cuban exports sector through the evolution of a group of indicators chosen in the revolutionary times, from 1959 to 2013 , in order to identify the main vulnerabilities it has to face in the short and middle terms. A descriptive historical approach has been taken based on the evolution of economical variables through that period (1959-2013). It was observed that the Cuban export sector has gone through important changes, keeping nonetheless structural weaknesses all along the studied period. From 1989 on Cuba had to adjust its trading relations due to the unexpected fall of the European socialist regimes with which it shared privileged relationships. In the following stage, trade has diversified, 
but the dependent relation with ideologically similar countries has kept on, as well as the above-mentioned weaknesses, that are far from facing an encouraging picture in the short run.

Key words: Cuban export sector; diversification; opening rate; Cuban economy.

Fecha de recepción: 18 de febrero de 2016. Fecha de aceptación: 26 de mayo de 2016.

\section{INTRODUCCIÓN}

lo largo de más de 55 años la economía cubana ha debido reestructurar su sector exterior al menos en dos periodos: 1959-1961 1 y 1990-1993. La adaptación a nuevos entornos internacionales ha implicado transformaciones estructurales, cambios en la política económica y costos de ajustes considerables (Quiñones y Rubiera, 2008). Es conocido que en el periodo histórico que analizamos (1959-2013), la economía cubana sufrió profundas transformaciones, tanto en las relaciones de propiedad, en su estructura económica y funcionamiento, como en las relaciones de distribución, que afectaron su sector externo y en general sus relaciones comerciales y financieras que la han vinculado con el mundo exterior.

Las transformaciones a partir de 1959-1960 en el sector exterior tuvieron que ver con la reorientación de sus mercados externos, por consiguiente con los socios comerciales y con la sustitución de los mercados del continente americano (como principales destino y origen de los bienes, servicios, tecnología y financiamiento necesario) por los mercados de Europa del Este y la antigua Unión Soviética, además de su participación en nuevos organismos internacionales de regulación del comercio y financiamiento exterior (Consejo de Ayuda Mutua Económica, CAME). Sin embargo no hubo cambios significativos en la estructura de su comercio exterior y en general en su perfil de especialización externa, basado en la exportación de azúcar de caña y de otros productos primarios como tabaco, cítricos o níquel.

Desde inicios de los años noventa, con la descomposición del sistema de relaciones económicas, comerciales, financieras que integraban a los países socialistas y en las cuales estuvo incluida la economía cubana durante tres décadas, comienza una etapa de crisis profunda -llamada periodo especial- y de transformaciones internas que afectaron el sector exterior. Entre los principales cambios están: la variación en la composición y la estructura del comercio exterior y del papel del azúcar en el total de exportaciones, el incremento de la contribución de los servicios y la reorientación 
geográfica de su comercio exterior, al desplazarse nuevamente sus socios principales al hemisferio occidental.

Con un enfoque histórico del comercio exterior como base del análisis de los indicadores económicos, el trabajo está dividido en dos partes: la primera abarca el periodo de 1959-1989 y la segunda comprende desde 1990 hasta 2013. Para ambos periodos se construyeron indicadores que evaluaron la evolución y dinámica del sector exportador cubano: tasa de apertura externa, orientación geográfica, composición del comercio exterior, entre otros. El estudio se realizó con fuentes estadísticas de diferente origen, tanto nacionales como extranjeras. ${ }^{1}$ En ambos periodos la información es escasa, diversa y heterogénea, resultado de diferentes sistemas de contabilidad. Se obtuvo un perfil de inserción internacional de la economía en el que destaca un sistema de relaciones económicas condicionadas a acuerdos políticos, cuya duración depende de la estabilidad y de las condiciones económicas y políticas de los socios comerciales.

\section{EL PRIMER PERIODO: 1959-1989}

\section{Apertura externa}

Presentamos algunas características del sector exterior cubano que ponen de manifiesto su importancia creciente para orientar la dinámica económica y sus debilidades y que analizamos desde el punto de vista de la concentración-diversificación de sus exportaciones, tanto por producto como por área geográfica de destino. Empezamos mostrando la importancia que el sector exterior ha adquirido en este primer periodo que va desde el inicio del proceso revolucionario (1959) hasta la caída del muro de Berlín (1989), con la consiguiente ruptura de las relaciones comerciales con el bloque socialista. La gráfica 1 muestra la evolución de la tasa de apertura, medida como relación entre el intercambio comercial y el producto social global (en adelante PSG). ${ }^{2}$

\footnotetext{
${ }^{1}$ Las particularidades de las estadísticas cubanas han sido expuestas por diversos economistas. Carmelo Mesa Lago (1969, 2012), reconocido académico, es quien más ha trabajado en el análisis y crítica de estas. Para el caso particular que nos ocupa, la información acerca del sector exportador de bienes y servicios que aparece en las fuentes directamente cubanas es limitada (Oficina Nacional de Estadística), por lo que en este trabajo se ha recurrido a otras fuentes para enriquecer la exposición. En el segundo periodo analizado no coinciden las cifras de las fuentes utilizadas (Oficina Nacional de Estadística y BACI-UN COMTRADE), por lo que para analizar las composición de las exportaciones se usaron las dos fuentes mencionadas.

${ }^{2}$ El PSG es una medida de la riqueza nacional que emplearon los países socialistas basándose en un sistema de planificación central. A partir de 1962 Cuba sustituyó el sistema de cuentas nacionales (en adelante $\mathrm{SCN}$ ) por el sistema del producto material (SPM) em-
} 


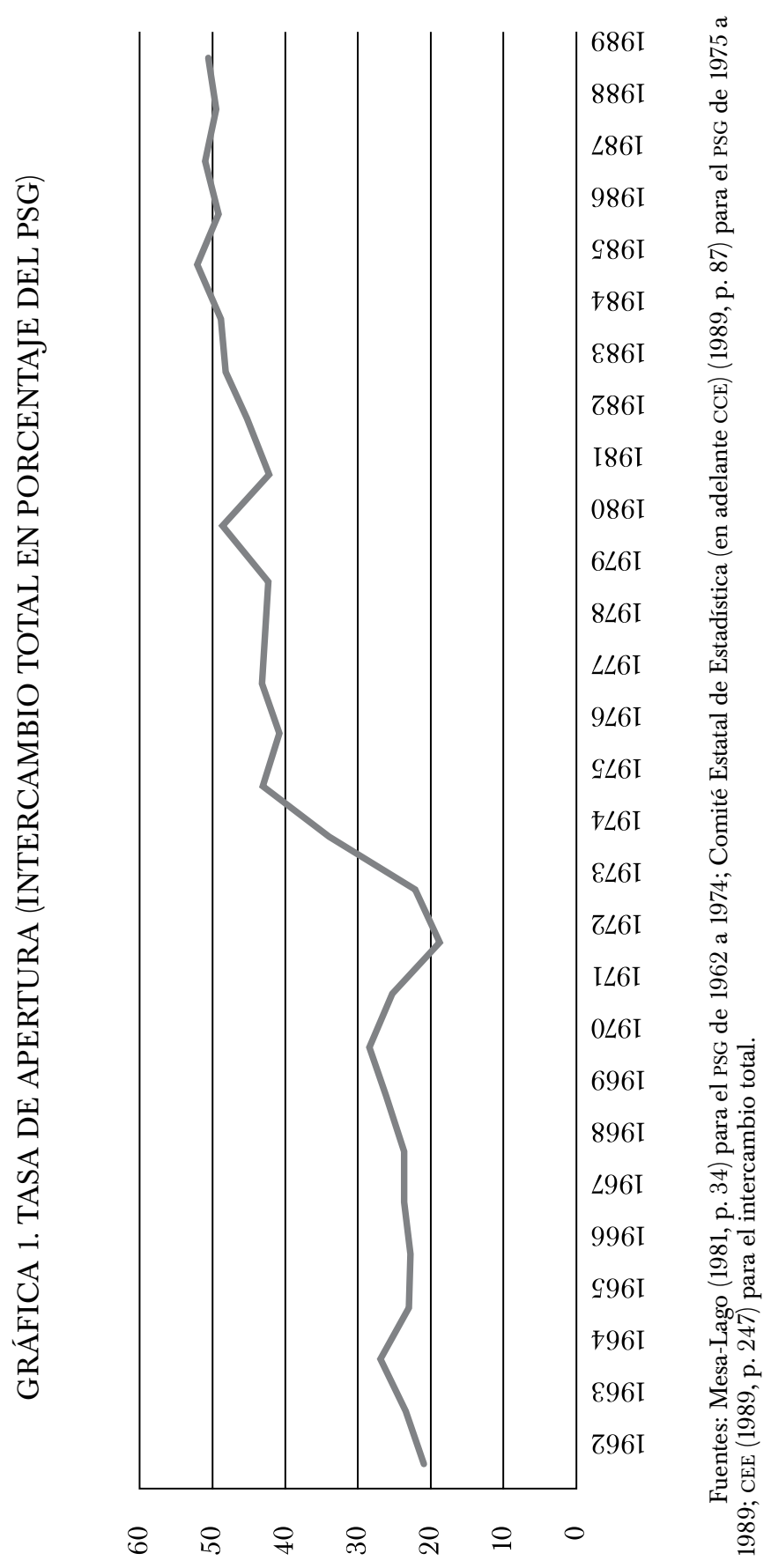


El indicador se mantiene entre 20 y $30 \%$ en los años sesenta, crece netamente entre 1973 y 1975, y luego conserva una tendencia creciente hasta 1989. El incremento del precio del azúcar en el mercado libre en 1973, que se multiplica por tres en un solo año, y el cambio metodológico en 1977 (Mesa-Lago y Pérez, 1985, p. 11), con el cual se reducen los dobles conteos en el PSG, son dos factores exógenos que contribuyen a causar el fuerte aumento entre 1972 y 1975. Sin embargo se pueden apreciar dos etapas: en la primera (hasta 1972) el índice se mantiene bajo y estable; en la segunda se multiplica por dos, y muestra una tendencia creciente. Esto se explica con el proceso de integración en el CAME que comenzó precisamente en 1972, la autocrítica del gobierno cubano sobre la etapa "idealista" de los años sesenta, y la alineación al bloque socialista guiado por la Unión Soviética del cual Cuba había tratado de prescindir en la primera década.

\section{Concentración geográfica}

El siguiente indicador mide, en este primer periodo, la concentración del intercambio comercial de la isla con el país líder del bloque socialista: la ex Unión Soviética. Después del aumento entre 1960-1961 (cuando Cuba tuvo que enfrentar la imprevista interrupción de las relaciones económicas con Estados Unidos), la cuota de intercambio comercial con el principal país socialista se mantuvo constante hasta 1974, luego volvió a crecer, al pasar de un valor promedio de $45 \%$ entre 1960 y 1975 , a $66 \%$ entre 1976 y 1989. En algunos años $(1963,1974,1980)$ la reducción del indicador coincide con el aumento del precio del azúcar en el mercado mundial, lo cual podría significar que en estos años Cuba, por conveniencia, desplazó sus ventas al mercado mundial y redujo las entregas a los soviéticos (gráfica 2).

\section{Composición y diversificación de las exportaciones (propuesta de método de cálculo del peso del azúcar en las exportaciones totales)}

Entre los indicadores más importantes del grado de dependencia de una economía se encuentra la composición y la diversificación de sus expor-

\footnotetext{
pleado en los países socialistas para medir el PSG. El PSG y el PIB se diferencian en que: a) el SCN incluye en el PIB el valor de todos los servicios. El PSG no contabiliza los llamados "servicios no productivos", como los sociales (educación, salud, seguridad social, vivienda), la defensa y ciertas actividades burocráticas. Por consiguiente, el PSG es menor que el PIB. b) El SCN utiliza el "valor agregado" en el cálculo del PIB. El SPM no, lo cual generaba un doble conteo o sobreestimación. De esta manera, hasta 1990 la contabilidad nacional cubana utilizó el SCN, por lo que hasta 1989 se cuenta exclusivamente con el PSG.
} 


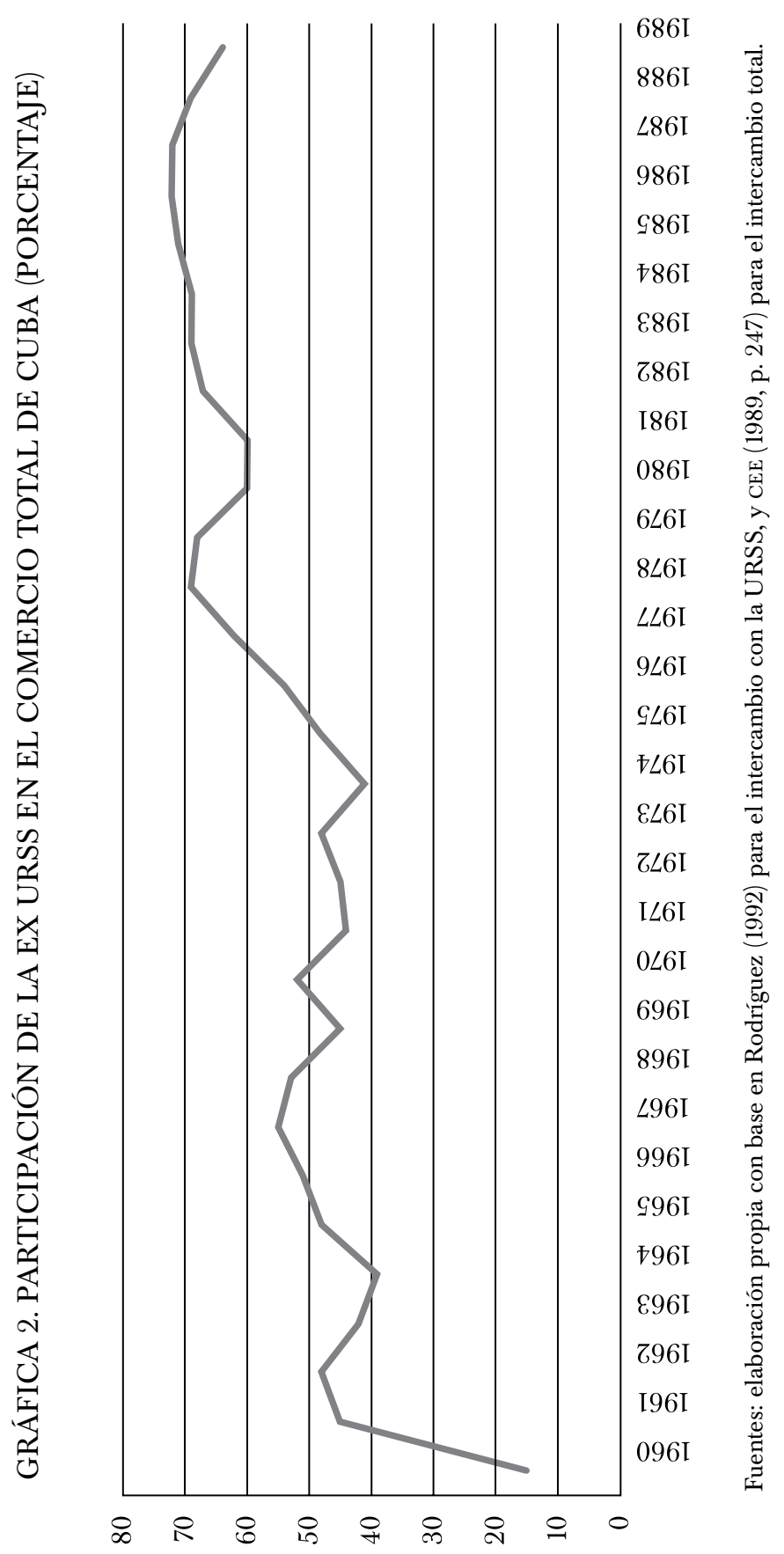


taciones. Cuanto menor sea la diversificación y tanto mayor la presencia de productos primarios entre las exportaciones, más dependiente será el país. En el caso de Cuba hubo varios intentos de comparar el peso del azúcar sobre las exportaciones totales antes y después de 1959, y la evolución del indicador en la etapa revolucionaria. Los estudios realizados no dan los mismos resultados; algunos padecen de errores metodológicos que señalamos y tratamos de corregir. Sin embargo hay cierto acuerdo en no reconocer importantes logros en esta dirección, a pesar de las intenciones declaradas por el gobierno de la isla.

El esfuerzo inicial para diversificar las exportaciones (primera estrategia de desarrollo) y librarse de la esclavitud de la caña, determinó una zafra desastrosa en 1963 (con una cosecha de 3880000 toneladas) y abrió un grave déficit en la balanza comercial. El año siguiente la estrategia de desarrollo se cambió radicalmente y el gobierno declaró como objetivo una zafra de 10000000 de toneladas para 1970. El cuadro 1, columna A, presenta la relación entre las exportaciones de azúcar y las exportaciones totales a precios corrientes.

De valores próximos a $80 \%$ en 1958, la relación entre el azúcar y las exportaciones a precios corrientes llega a $90 \%$ en 1975, luego empieza una disminución gradual. En los últimos años del periodo considerado alcanza su valor mínimo (73.4\%), lo que haría pensar en un éxito parcial y limitado de la política comercial cubana en relación con la diversificación de las exportaciones.

Sin embargo, este indicador presenta dos problemas: ${ }^{3}$ el primero incluye entre las exportaciones las reexportaciones de petróleo que no son un producto interno y causan un aumento del denominador, lo que reduce el índice; ${ }^{4}$ en el cuadro 1 se percibe (columna B) que cuando se eliminan las reexportaciones de petróleo de las exportaciones totales, la rápida disminución de los años ochenta apenas se percibe hasta 1986, y sólo a partir de 1987 el indicador se reduce por debajo de 80\%. El segundo problema es de más difícil solución, ya que obliga a considerar a precios constantes el indicador que queremos construir. ${ }^{5}$

\footnotetext{
${ }^{3}$ La tabla construida por Rodríguez (1988, p. 29) no toma en cuenta ninguno de estos dos problemas.

${ }^{4}$ Desde el punto de vista contable, como se expone más adelante, entre las exportaciones se debería registrar el área capitalista. Si medimos el grado de diversificación de las exportaciones, queda claro que la presencia de un producto importado de la Unión Soviética entre las exportaciones distorsiona hacia abajo la relación entre exportaciones de azúcar y exportaciones totales, sin que represente un proceso real de diversificación de las exportaciones. Por eso calculamos las exportaciones cubanas al neto de las reexportaciones de petróleo.

${ }^{5}$ Pérez (1991a, p. 212) observa que esto es muy importante porque la URSS pagaba precios muy superiores a los del mercado mundial.
} 


\section{CUADRO 1. EXPORTACIONES DE AZÚCAR SOBRE EL TOTAL (PORCENTAJE)}

\begin{tabular}{lllllll}
\hline Año & $(A)$ & $(B)$ & $(C)$ & $(D)$ & $(E)$ & $(F)$ \\
1958 & 79.7 & 79.7 & 79.7 & 83.4 & 83.5 & 70.9 \\
1965 & 85.9 & 85.9 & 85.9 & 91.4 & 91.4 & 84.5 \\
1970 & 76.8 & 76.8 & 76.8 & 90.6 & 90.6 & 83.5 \\
1975 & 90 & 90 & 90 & 89.1 & 89.1 & 79.6 \\
1980 & 83.9 & 86 & 86 & 85 & 85.1 & 73.6 \\
1981 & 79.2 & 82.2 & 82.2 & 87.1 & 87.1 & 78.1 \\
1982 & 77.3 & 81.6 & 81.6 & 86.6 & 86.6 & 77.9 \\
1983 & 74.1 & 81.4 & 81.3 & 84.3 & 84.3 & 74 \\
1984 & 75.4 & 82.7 & 82.3 & 84.9 & 84.9 & 76.1 \\
1985 & 74.6 & 81.8 & 81.4 & 84.3 & 84.3 & 73.8 \\
1986 & 77.2 & 80.9 & 80.5 & 83.2 & 83.3 & 72.1 \\
1987 & 74.4 & 78.6 & 77.8 & 82.4 & 82.4 & 72.3 \\
1988 & 74.7 & 77.3 & 76.4 & 84 & 84 & 71.8 \\
1989 & 73.4 & 76.2 & 74.5 & 83.2 & 83.1 & 72.3 \\
\hline
\end{tabular}

Nota: (A) variables medidas a precios corrientes; $(\mathrm{B})=(\mathrm{A})$ menos reexportaciones de petróleo; $(\mathrm{C})=(\mathrm{B})$ menos reexportaciones de azúcar; (D) variables medidas a precios de 1975 y con ponderaciones medias; $(\mathrm{E})$ variables medidas a precios de 1975 y con las ponderaciones de $1965 ;(\mathrm{F})$ variables medidas a precios de 1965 y ponderaciones de 1965.

Fuentes: elaboración propia con base en CEE, 1985 (pp. 396-405) y 1989 (pp. 264-273) para las exportaciones de azúcar y totales en pesos cubanos; Banco Nacional de Cuba (en adelante BNC) $(1986$, p. 23,1987 , p. 23,1988 , p. 15,1989 , p. 15,1990 , p. 22) para las reexportaciones de petróleo; BNC (1986 p. 28, 1987, p. 25, 1989, p. 12, 1990, p. 23) para las reexportaciones de azúcar.

Pérez (1991a, pp. 214-217) observa que es importante eliminar de las exportaciones de azúcar cubanas el subsidio pagado por los soviéticos. El autor calcula el valor de las exportaciones de azúcar a la Unión Soviética (Pérez, 1991a, p. 215) usando precios distintos de los que pagaban los soviéticos, luego determina el peso del azúcar sobre las exportaciones totales a precios corrientes, a partir de estos nuevos valores. Si este procedimiento puede considerarse un primer paso hacia la solución del problema, es sin embargo insuficiente y es necesario medir a precios constantes el indicador. ${ }^{6}$ Mientras que la valoración del numerador a precios constantes es

${ }^{6}$ El mismo Pérez (1991b) observa que "conceptualmente la medición a precios constantes que mantiene los precios de productos en la canasta de las exportaciones a los niveles de 1965, parece ser más apropiado para registrar el impacto de las fluctuaciones de los precios del mercado mundial sobre la estructura de la producción y de las exportaciones en lugar de los altos precios pagados por la Unión Soviética" (p. 38). 
inmediata, la medición de las exportaciones a precios constantes aparentemente se dificulta por la poca información disponible.

Zimbalist y Brundenius (1989, pp. 145-147) y Zimbalist (1990, pp. 133135) han realizado un intento serio de resolver el problema, con resultados muy contradictorios. En primer lugar Zimbalist utiliza 1965 como año base sobre el cual bajar los precios, sin una razón clara. En ese año el precio del azúcar en el mercado mundial fue de sólo dos centavos la libra (uno de los más bajos de todo el periodo), mientras que el precio pagado por los soviéticos fue netamente inferior al promedio del periodo, lo cual contribuye a sesgar el índice final. Zimbalist (1990) afirma que el cálculo en precios constantes (de 1965) de las exportaciones "requiere la construcción de un índice de precios de las exportaciones sin una completa información sobre los precios" (p. 133). El autor propone la construcción de un índice de precios con base en una información contenida en el Anuario Estadístico de $C u b a$ (en adelante $A E C$ ) de $1985,^{7}$ en la cual las exportaciones cubanas se dividen en seis categorías. Zimbalist (1990) observa que en cada una de estas categorías:

se incluyen los principales productos de exportación con datos sobre los volúmenes y las ventas en los años 1965, 1970, 1975, 1980, 1985. Por tanto se pueden calcular en cada año los precios promedio a la exportación y se pueden construir los índices de precio para cada categoría. El problema luego es cómo agregar los índices de las categorías en un solo índice global de precios de las exportaciones. Hemos agregado usando las cuotas en valor tanto de 1965 como de 1985 (a precios corrientes), de cada categoría sobre el total de las exportaciones. En cuanto los precios del azúcar crecen más rápidamente en el periodo 1965-1985 que los precios de las otras exportaciones, y considerando que la cuota de azúcar (a precios corrientes) sobre las exportaciones era mayor en 1965 (85.8\%) que en 1985 (74.4\%), usando las cuotas en valor de 1965 aumenta más rápidamente el deflactor del precio de las exportaciones. Entonces usando los pesos de 1985 lleva a un aumento de las exportaciones totales a precios constantes mayor que cuando usamos los pesos de 1965 y una estimación menor de la cuota de azúcar sobre el total de las exportaciones en 1985 (pp. 133-134).

En realidad Zimbalist no se da cuenta de que, sumando cada producto registrado en el $A E C$ para el cual hay informaciones sobre el valor y la cantidad exportada (por lo que se puede determinar directamente el precio unitario), se cubre todo el valor de las exportaciones hasta 1975 (incluido).

\footnotetext{
${ }^{7}$ También en el Anuario Estadístico de Cuba de 1989 aparece esa información, actualizada a este año. Es probable que no hubiera sido publicada todavía cuando Zimbalist entregó el artículo para publicación (CEE, 1989).
} 
El cuadro 2 muestra que de los primeros años sólo en 1965 el peso de las exportaciones, de las cuales sólo tenemos el dato en valor sobre el total de las exportaciones, es superior a $1 \%(1.1 \%)$, mientras en 1970 era de $0.84 \%$, y de $0.54 \%$ para 1975 , y aumentó en 1980 . Sin embargo, si restamos a las exportaciones totales las reexportaciones de petróleo, el índice se mantiene en valores muy bajos (entre 2 y 4\%) por todo el periodo 1980-1986 para acercarse a 5\% en 1987. Esto significa que la construcción del índice de precios propuesta por Zimbalist en algunos años es prácticamente innecesaria $(1965,1970,1975)$, y en los otros se tendría que aplicar a una cuota muy baja de exportaciones para las cuales sólo conocemos el dato en valor $\mathrm{y}$ que tienen que ser deflactadas con un índice de precio.

Se trata de una observación esencial para delimitar y prácticamente eliminar el problema teórico que con razón Zimbalist considera no solucionable, es decir el de la elección de los pesos con los cuales ponderar los índices de precio de cada categoría de exportaciones para llegar a un índice global de precios de las exportaciones.

Las columnas A y B del cuadro 3, adaptado del de Zimbalist (1990, p. 134), muestran resultados muy distintos: la primera columna está construida con las cuotas de 1965, la segunda con las de 1985 .

Según la primera columna, un proceso de diversificación de las exportaciones comienza a partir de 1980; sin embargo en 1985 las exportaciones de azúcar siguen siendo $74.6 \%$ del total. Si usamos las cuotas de 1985, la diversificación empezaría ya en los años setenta y seguiría en la década de los ochenta, con el índice que baja a 64.3\% para 1985.

Zimbalist (1990) concluye que "desafortunadamente ningún deflactor es teóricamente más correcto que el otro", y añade que "una técnica con frecuencia usada en estos casos es la de considerar el promedio de los resultados de los dos métodos" (p. 135).

A continuación se presenta la propuesta del método para medir el peso del azúcar sobre el total de las exportaciones de Cuba. En primer lugar se escogió 1975 como año base, sobre el cual deflactar, por dos razones: $a$ ) en ese año el precio pagado por la Unión Soviética se acerca mucho al del mercado mundial, lo cual reduce la distorsión que se crea al sumar el valor del azúcar vendido al CAME (en gran prevalencia a la URSS) y a las economías de mercado, utilizando para la conversión la tasa de cambio oficial, ya que el precio que aparece en el $A E C(1989)$ es el global; $\left.{ }^{8} b\right)$ en 1975 el precio del azúcar pagado por los soviéticos no es tan distinto del precio promedio en todo el periodo.

\footnotetext{
${ }^{8}$ En el $A E C(1989)$ la venta total de azúcar es la suma de las ventas en moneda convertible y al CAME, ambas convertidas en pesos cubanos con la tasa de cambio oficial.
} 
CUADRO 2. EXPORTACIONES NO REGISTRADAS (PORCENTAJE DEL TOTAL)

\begin{tabular}{lrl}
\hline Año & $(A)$ & $(B)$ \\
& & \\
1958 & 8.04 & 8.04 \\
1965 & 1.11 & 1.11 \\
1970 & 0.84 & 0.84 \\
1975 & 0.53 & 0.53 \\
1980 & 3.82 & 1.34 \\
1981 & 5.91 & 2.41 \\
1982 & 7.78 & 2.61 \\
1983 & 12.01 & 3.32 \\
1984 & 12.33 & 3.82 \\
1985 & 12.18 & 3.71 \\
1986 & 8.41 & 3.93 \\
1987 & 9.93 & 4.89 \\
1988 & 9.67 & 6.54 \\
1989 & 11.99 & 8.69 \\
\hline
\end{tabular}

Nota: (A) Incluyendo todas las exportaciones; (B) excluyendo las reexportaciones de petróleo.

Fuentes: elaboración propia con base en CEE (1989, pp. 264-273); BNC (1986, p. 23, 1987, p. 23, 1988, p. 15, 1989, p. 15, 1990, p. 22).

CUADRO 3. EXPORTACIONES DE AZÚCAR SOBRE EL TOTAL (VALORES EN PORCENTAJE)

\begin{tabular}{lcc}
\hline$A \tilde{n} o$ & $(A)$ & $(B)$ \\
& & \\
1965 & 84.5 & - \\
1970 & 86.7 & 83.8 \\
1975 & 82.2 & 76.2 \\
1980 & 89.1 & 74.8 \\
1985 & 74.6 & 64.3 \\
\hline
\end{tabular}

Nota: (A) utilizando las cuotas de 1965; (B) utilizando las cuotas de 1985.

Fuente: adaptado de Zimbalist (1990, p. 134). 
Una posible objeción sería que en este año el precio del mercado mundial era muy distinto del promedio en el periodo. Sin embargo, cabe aclarar que las ventas del azúcar cubano en el mercado mundial eran de un tercio del total y que el precio de 1975 queda lejos de los valores más altos de 1974 y 1980, y también de los valores muy bajos de los primeros años. Aun así, la elección del año base es discrecional; por tanto nos pareció oportuno considerar también 1965 como año base, lo cual permite, entre otras cosas, una comparación más directa con los datos de Zimbalist.

En segundo lugar, no se han usado las cuotas de ningún año en específico. Tratándose de una decisión discrecional, se considera que se reducen los efectos distorsionantes sobre los resultados finales tomando el promedio en todo el periodo, en lugar de considerar sólo el año inicial y el final como en Zimbalist. Para completar también se hicieron los cálculos con pesos de 1965.

Las distintas fases de la determinación, a precios constantes de 1975, del valor de las otras exportaciones para las cuales se dispone del solo dato en valor, obtenidas a precios corrientes por diferencia entre las exportaciones totales y la suma de las que están en el $A E C(1989)$ en cantidad y valor, son: a) el cálculo del promedio de la composición de las exportaciones registradas en el $A E C$ (a precios corrientes), o sea su peso promedio; $b$ ) la determinación por cada año de la tasa de variación sobre el año base del precio correspondientes a cada una de las exportaciones que aparecen en el $A E C$, en cantidad y valor y para las cuales es inmediato el cálculo de este precio; $c$ ) la ponderación de cada precio por los pesos determinados en el punto uno, y d) la suma de estos últimos, para determinar el deflactor que se aplica a todas las exportaciones en los distintos años, cuyo valor se obtiene a precios constantes de 1975 .

Con excepción de 1958 -año en el que para 8\% de las exportaciones no se puede construir el índice de precios de las exportaciones y para el cual es necesario deflactar-, en 1965, 1970 y 1975 las exportaciones cuyo cálculo a precios constantes necesita del procedimiento descrito son, respectivamente, 1.1, 0.08 y $0.05 \%$ del total. Los resultados obtenidos, después de haber deflactado sobre 1975 las exportaciones registradas sólo en valor, son totalmente correctos para estos años y muestran que hasta el primer quinquenio de los años setenta no hubo ninguna diversificación apreciable, lo que rechaza la segunda columna de la tabla de Zimbalist. Según nuestros datos, es mucho menos importante la diversificación de las exportaciones que muestran ambas columnas en la tabla de Zimbalist del cuadro 3, y que se acentúa en la década de los ochenta

En 1981 el valor en precios de 1975 del azúcar sobre el total de las exportaciones es todavía superior a $87 \%$ y desde entonces comienza una lenta disminución, con el indicador que en 1989 todavía está a 83.1 por ciento. 
Una importante prueba de la eficacia de nuestro método es que, cambiando los pesos y utilizando los de 1965 en lugar de los valores promedio como en el primer caso, se obtienen valores idénticos para 1965, 1970, 1975 como era lógico esperar, pues en esos años las exportaciones que tenían que ser deflactadas no eran significativas como hemos visto. Pero también el valor promedio en la década de los ochenta es independiente de las ponderaciones utilizadas. Esto es muy significativo, ya que el problema teórico que Zimbalist consideraba "no solucionable" y que lo llevaba a datos muy distintos, sin tener un criterio para escoger la mejor estimación, no se elimina con nuestro procedimiento, pero se anulan prácticamente los efectos distorsionantes sobre los datos finales. Por tanto, los resultados de nuestra estimación dependen muy poco de los cambios en los pesos utilizados, lo cual los hace muy confiables.

Aplicamos el mismo criterio tomando 1965 como base (cuadro 1, columna F), y se obtuvieron los siguientes resultados: en los últimos años el indicador vuelve a 70\%, que era su valor en 1958; en la década de los setenta hay una tendencia a la disminución del indicador que sin embargo se mantiene en toda la década de los ochenta en los valores de 1980, lo que confirma nuestro primer cuadro y refuta los datos de Zimbalist; mientras que las tendencias detectadas cuando el año base era 1975 se confirman, con los nuevos precios el indicador es menor en términos absolutos en promedio de diez puntos porcentuales.

Como decíamos inicialmente, nos parece que hay más razones para utilizar 1975 como base, lo cual nos induce a considerar más correctas las columnas D y E del cuadro 1. No queda claro cómo comportarse con las reexportaciones de azúcar, pues, al igual que las reexportaciones de petróleo, tampoco se producen internamente. Sin embargo, puesto que repercuten casi de la misma manera tanto en el numerador como en el denominador del indicador, no llevan a distorsionar los datos, como muestra la ligera diferencia entre las columnas $\mathrm{B}$ y $\mathrm{C}$ del cuadro 1 , que considera los dos casos a partir de 1982, cuando aparecen por primera vez estas reexportaciones.

Según un estudio de la Conferencia de las Naciones Unidas sobre Comercio y Desarrollo (UNCTAD, por sus siglas en inglés) documentado por Pérez (1991a, p. 212), en 1960 Cuba se encontraba en los últimos lugares en cuanto al grado de diversificación de sus exportaciones, y fue el único país cuya situación no mejoró en el periodo de 1960-1985. Eso es tanto más grave si se considera que el azúcar es, entre los productos primarios, el que muestra la mayor volatilidad en los precios. ${ }^{9}$

\footnotetext{
${ }^{9}$ Fry y Tsadik en Pérez (1991a, pp. 124-125) identifican dos causas: a) las diferencias entre demanda y oferta sólo repercuten en el mercado "libre", por tanto se multiplican allí sus efectos;
} 
Hemos analizado la diversificación de las exportaciones en la época revolucionaria, además de mostrar y tratar de solucionar algunos de los problemas que se plantean, pero no hemos examinado todavía el que consideramos más importante en términos teóricos: el de la necesidad de sumar datos en moneda convertible y en rublos no transferibles. Las estadísticas cubanas no hacen esta diferencia y agregan todas las exportaciones utilizando la tasa de cambio oficial, que, como explicaremos más adelante, resulta sobrevalorada, y en la transformación en pesos cubanos de los rublos no transferibles, económicamente no significativa. En el caso del azúcar, por ejemplo, se utilizan mediciones a precios muy distintos -sobre todo a partir de 1982-, lo que distorsiona resultados y conclusiones. Sin embargo, en este caso los resultados no pierden totalmente su significado, máxime por la elección de un año base (1975) en el que el precio del azúcar pagado por los soviéticos y en el mercado libre (con la conversión al tipo de cambio oficial) son muy cercanos.

\section{Comparación con el periodo prerrevolucionario}

Comparamos ahora los indicadores que hemos construido para el periodo revolucionario con un valor promedio antes de 1959. Además de los indicadores considerados hasta ahora (apertura externa, concentración del comercio con un solo país, grado de diversificación de las exportaciones), hemos construido otros dos: el intercambio comercial sobre el PIB y el de las exportaciones de azúcar (siempre sobre el PIB).

Un primer problema es construir el PIB en el periodo revolucionario, ya que no aparece en la contabilidad socialista, donde no se consideran los servicios no materiales. El indicador que más se le acerca es el INC (ingreso nacional creado) que evita, al menos teóricamente, los dobles conteos, pero no considera los servicios no materiales. Se estimaron los servicios no materiales, usando como proxy de su peso sobre el INC la relación entre los pagos a los sectores no productivos sobre el total. Al respecto, Mesa-Lago y Pérez (1985) correctamente observan que los datos oficiales no comprenden los pagos al sector privado, al ejército y a los servicios secretos; sin

\footnotetext{
b) una parte importante del azúcar que se exporta en el mercado libre es de caña, cuya oferta es poco elástica al precio, ya que se requieren de uno a dos años para que una nueva planta llegue a maduración. $\mathrm{Si}$, por ejemplo, se crea un exceso de demanda que las cantidades almacenadas no pueden satisfacer, la mayor cantidad estará disponible cuando la situación en el mercado sea distinta.
} 
embargo, como ellos mismos reconocen, su método tampoco puede ser exento de críticas. ${ }^{10}$

Después de haber obtenido una estimación del PIB (véase cuadro 4), ${ }^{11}$ pasamos a construir los indicadores mencionados.

En el cuadro 5 se puede observar que ningún indicador muestra una clara mejoría antes de 1989, o sea una menor dependencia, en relación con la situación antes de 1959. Sólo el índice Galtung (véase anexo 1) parece evolucionar favorablemente, pero su disminución en valor absoluto se debe no tanto a que aparezcan bienes manufactureros entre las exportaciones, sino al aumento en la importación de bienes primarios (sobre todo el petróleo).

Podemos concluir que las debilidades estructurales del sector exportador de la economía cubana en la época prerrevolucionaria (concentración del intercambio con un solo gran país, exportación sólo de bienes primarios y peso excesivo del azúcar sobre las exportaciones) se mantuvieron hasta 1989, como se analizará en la siguiente sección, a pesar de los profundos cambios que introdujo el gobierno revolucionario en las relaciones comerciales y en la estructura productiva.

\section{Segundo periodo: 1990-2013}

El año 1990 marca un periodo completamente nuevo. La desaparición del socialismo y de los acuerdos preferenciales que la isla tenía para el acceso a los mercados de Europa del Este y la antigua URSS sirvieron de detonante de la crisis económica, cuya profundidad y consecuencias no se consideran superadas completamente por algunos autores (García Fernández, López y Sovilla, 2010; Nova, 2015). Esta nueva situación afectó obviamente el comercio exterior y en general el modelo de inserción internacional de la economía cubana.

\footnotetext{
${ }^{10}$ Mesa-Lago y Pérez (1985, p. 35) observan una gran diferencia entre el valor de la relación entre servicios no productivos y PIB que ellos mismo estiman a partir de los datos reportados en el presupuesto público y el único dato oficial de parte cubana. Ellos concluyen que la gran diferencia entre los dos resultados "pone en duda el utilizo de los datos sobre los gastos del presupuesto público como proxy del valor de los servicios no materiales".

${ }^{11}$ También nuestra estimación del PIB en el periodo revolucionario es discutible. Sin embargo, se trata de un paso adelante respecto, por ejemplo, a la comparación de LeoGrande (1979, p. 16) entre variables tan distintas como el PIB y el INC.
} 


\section{CUADRO 4. ESTIMACIÓN DEL PIB A PARTIR DEL INC}

\begin{tabular}{llrrl}
\hline$A \tilde{n} o$ & $(A)=x$ & $I N C$ & $P I B^{\mathrm{a}}$ & $(B)$ \\
1978 & 26.7 & 9987 & 12649 & 55.4 \\
1979 & 27.6 & 10051 & 12828 & 56 \\
1980 & 28.5 & 9523 & 12241 & 70.2 \\
1981 & 28.2 & 11504 & 14748 & 63.3 \\
1982 & 29.1 & 12087 & 15601 & 67.1 \\
1983 & 29.3 & 12745 & 16483 & 71.3 \\
1984 & 29.4 & 13696 & 17722 & 71.7 \\
1985 & 30 & 14261 & 18538 & 70.7 \\
1986 & 30.6 & 13944 & 18207 & 74.6 \\
1987 & 31.1 & 13273 & 17397 & 74.2 \\
1988 & 30 & 13565 & 1764 & 77 \\
1989 & 30.1 & 13496 & 17559 & \\
\hline
\end{tabular}

${ }^{\mathrm{a}} \mathrm{PIB}=\mathrm{INC}(1+\mathrm{x})$ (millones de pesos); (A) pagos al sector no productivo sobre los pagos totales (porcentaje); (B) intercambio comercial sobre el PIB (porcentaje).

Fuente: elaboración propia con base en CEE (1989, pp. 82, 113).

\section{CUADRO 5. DEPENDENCIA ECONÓMICA (ANTES Y DESPUÉS DE 1959)}

Cuba-Estados Unidos

(antes de 1959)

(A) Intercambio/PIB

(B) Exportaciones de azúcar/PIB

(C) Índice Galtung

(D) Comercio con un solo país/total

(E) Exportaciones de azúcar/total de exportaciones
56

26

$-0.55$

69

85
Cuba-URSS

(hasta 1989)

Nota: (A) valores promedio 1946-1958 y 1976-1989; (B) valores promedio 1947-1958 y 19801989; (C) valores promedio 1947-1958 y 1980-1989; (D) valores promedio 1946-1958 y 1976-1989; (E) valores promedio 1947-1958 y 1980-1989.

Fuentes: antes de 1958, LeoGrande (1979, pp. 8-15); para el periodo revolucionario son promedios de los indicadores de las tablas 1 y 2 . 


\section{Apertura externa}

El shock externo causado por la contracción absoluta del comercio exterior, entre 1991 y 1994, redujo la apertura de la economía (véase gráfica 3). En los años mencionados, el intercambio externo se contrajo 74\% (Oficina Nacional de Estadística [ONE], 2000), expresión de la reducción absoluta de las exportaciones e importaciones simultáneamente, en tanto que el PIB, se redujo 34\%, medido a partir de las cifras oficiales del Banco Nacional de Cuba (BNC, 1994). El efecto combinado de la reducción del intercambio externo $(\mathrm{X}+\mathrm{M})$ y del PIB (numerador y denominador al mismo tiempo) provocó que la reducción de la apertura no fuera mayor, pues la reducción del PIB atenuó la caída de la apertura. A partir de 2004 la apertura externa se incrementó y llegó a cerca de 50\% debido al incremento sobre todo de la exportación de servicios profesionales, pues la exportación de bienes no ha aumentado significativamente en relación con años anteriores.

\section{Orientación geográfica}

Hasta 1990 el comercio exterior cubano estuvo altamente concentrado en Europa y en particular en Europa del Este y la ex Unión Soviética. A partir de entonces, tiene lugar la reorientación geográfica del comercio exterior, pues en 1995 entre Europa y América se repartían en igual medida 80\% de todo el comercio exterior cubano (véase cuadro 6). El comercio exterior cubano comienza un proceso de desconcentración al adquirir cada vez más importancia su mercado natural, el continente americano, y dentro de él, América Latina. Ese proceso continúa hasta la actualidad, ya que en 2013 Europa del Este absorbió sólo 2\% de su intercambio comercial, mientras en 1990 captaba más de las tres cuartas partes del intercambio total (véase cuadro 6).

Por países, en 1994 la Federación Rusa era el destino de 20\% de las exportaciones y el origen de sólo $2 \%$ de las importaciones, lo que indicaba claramente que ese mercado dejaba de tener importancia para la economía -como fuente de materias primas y de otros bienes y servicios-, con las consecuencias que eso implicaba para el aparato productivo de la isla (ONE, 2000). Con la excepción del año 1995, la Federación Rusa se mantuvo como principal destino de las exportaciones cubanas hasta 2001, pero a partir del año siguiente dejó de ocupar esta posición y desapareció de los cinco primeros destinos en el año 2005 (ONE, 2000) (véase gráfica 4). A partir de 2004, con el incremento de la exportación de servicios, Venezuela se convirtió en uno de los principales socios comerciales (tercero) y desde 2009 ocupa el primer lugar (Oficina Nacional de Estadística e Información [ONEI], 2012). 


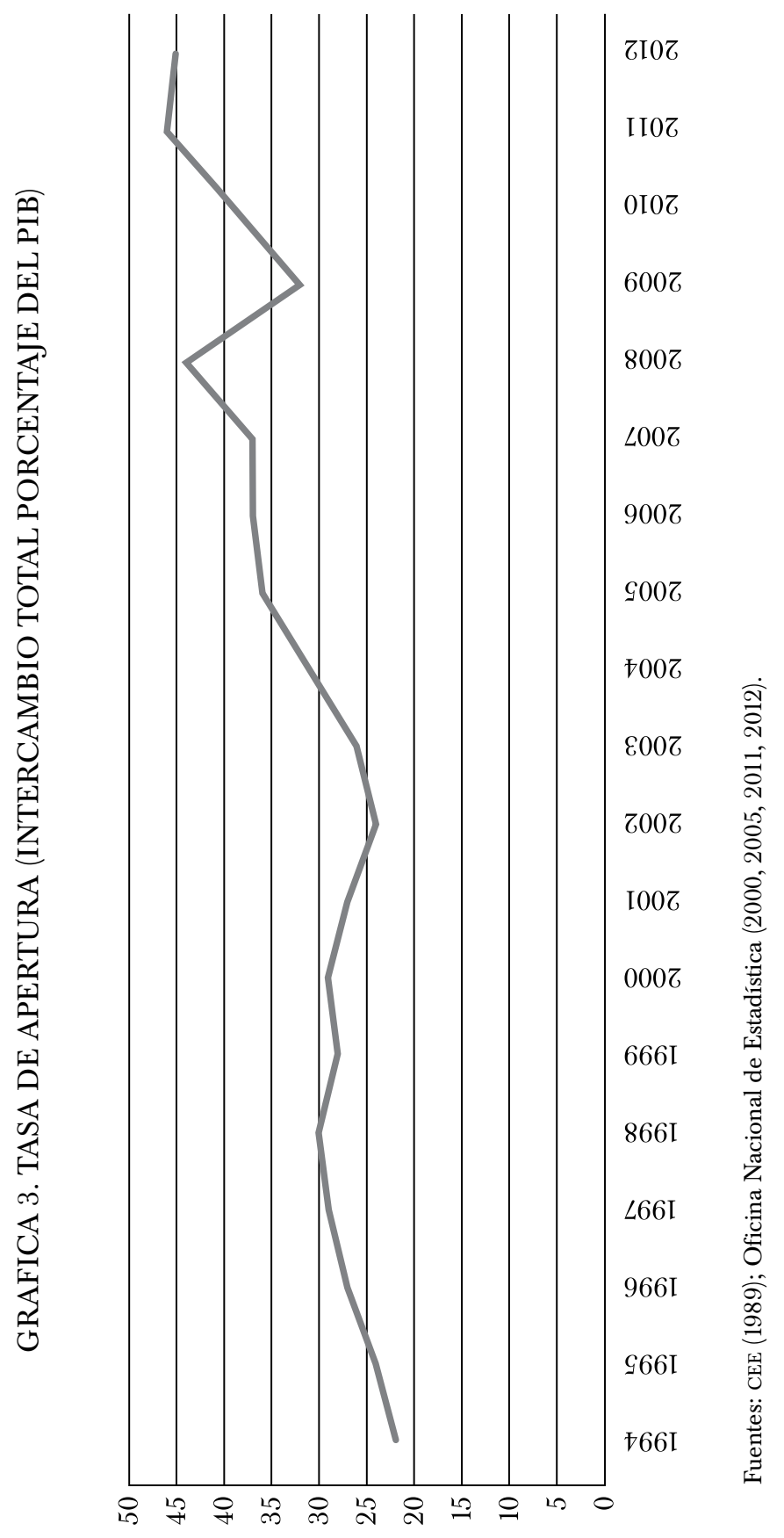




\section{CUADRO 6. ORIENTACIÓN GEOGRÁFICA DEL COMERCIO EXTERIOR (PORCENTAJE): 1990-2013}

\begin{tabular}{lccccccc}
\hline & 1990 & 1995 & 2000 & 2005 & 2010 & 2012 & 2013 \\
& & & & & & & \\
Europa & 84.9 & 41.6 & 43.3 & 29.9 & 19.8 & 21.8 & 24.43 \\
Europa del Este & 76 & 9 & 7.8 & 3.2 & 2.66 & 3.25 & 1.19 \\
América & 6.5 & 41 & 40.9 & 49.15 & 58.7 & 63.03 & 62 \\
América Latina & 5.7 & 30.7 & 31.8 & 36.1 & 49.93 & 55.56 & 53.24 \\
Asia & 6.8 & 14.1 & 13.7 & 18 & 18.3 & 12.38 & 12.06 \\
Total & 100 & 100 & 100 & 100 & 100 & 100 & 100 \\
\hline
\end{tabular}

Fuentes: Mañalich (2004) hasta año 2000. Elaboración propia a partir de 2005 (Oficina Nacional de Estadística e Información, 2005, 2012, 2014).

\section{Composición y diversificación de las exportaciones}

Con la desaparición del socialismo en países de Europa del Este y la Unión Soviética, la economía cubana tuvo que reconfigurar su comercio exterior, pues desaparecieron los mercados de sus principales productos de exportación e importación de materias primas, alimentos, maquinarias, tecnologías y de subsidios (Mesa-Lago, 2013; García Fernández et al., 2010). La nueva situación del país (ausencia de mercados garantizados para sus bienes de exportación, proveedores de materias primas, energía, tecnologías y bienes de consumo, para abastecer el mercado interno y de fuentes de financiamiento) forzó al gobierno cubano, después de casi cuatro años de inmovilismo en políticas económicas (1990-mediados de 1993), a instrumentar una serie de medidas para intentar paliar la grave crisis del país y reconfigurar su sector exterior. Este proceso abarcó dos etapas. La primera, a partir de mediados de 1993 y hasta aproximadamente 1996-1997; la segunda, desde 2009 hasta la actualidad, cuando Raúl Castro asume la conducción del gobierno con un paquete de reformas económicas e institucionales que se han ido estableciendo lentamente durante los años posteriores, hasta la nueva Ley de Inversión Extranjera (marzo 2014).

Precisamente como resultado de la crisis económica y de las medidas de política económica que se aplican, el sector exterior va sufriendo en los años noventa y 2000 una transformación estructural. Algunos de los cambios más importantes fueron: la incorporación al flujo internacional de remesas familiares y en menor medida de inversión extranjera directa; la reorientación del comercio exterior desde Europa del Este y la antigua Unión Soviética, hacia el continente americano y China (exportando a 


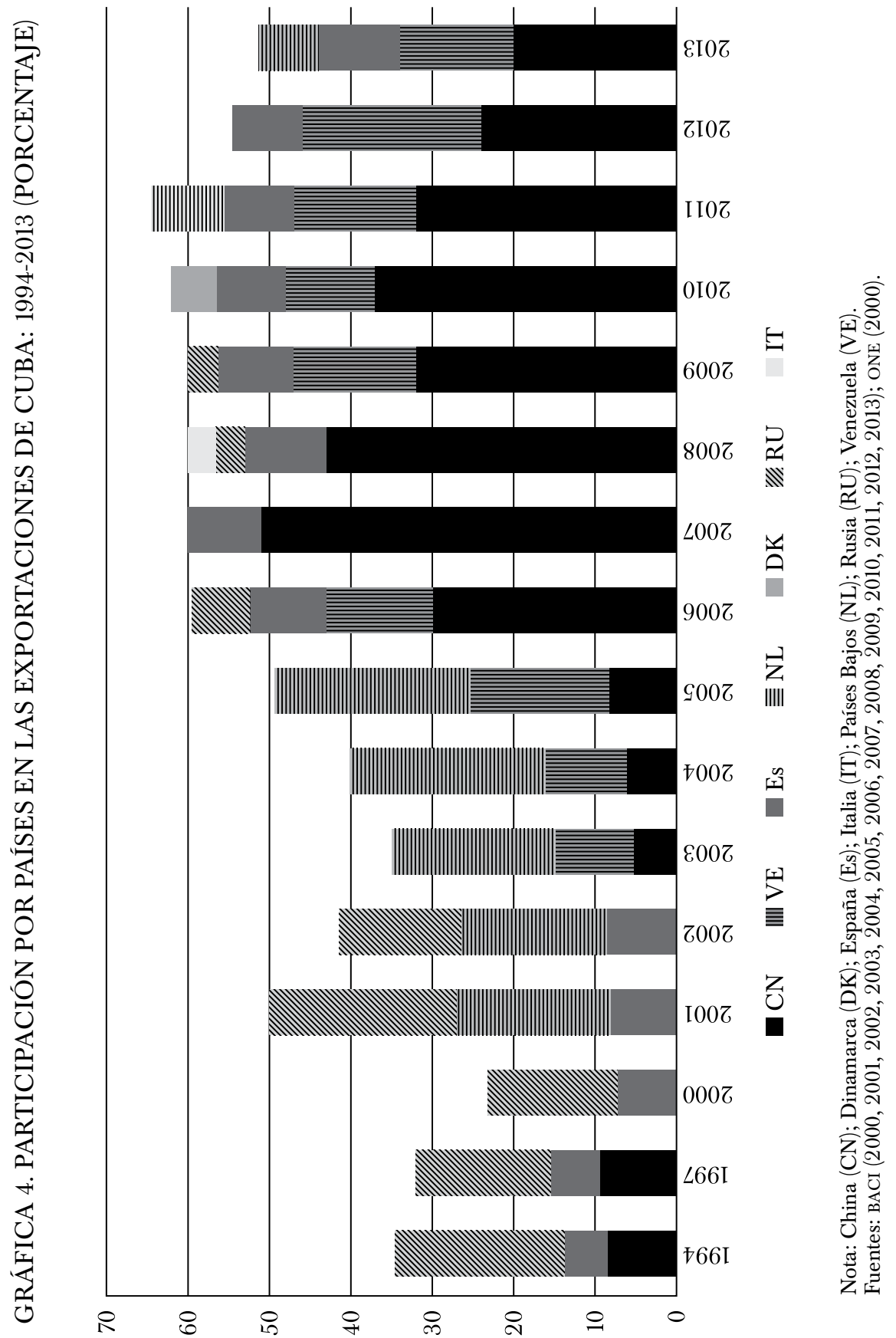


este níquel y azúcar e importando productos manufacturados) y la participación en esquemas de integración regional, como el de la Alianza Bolivariana (en adelante ALBA); el cambio en el perfil de especialización externa de la economía cubana, que pasó de una economía azucarera a una especializada en servicios, fundamentalmente en turismo y en exportación de trabajo profesional en las áreas de salud, educación y deportes.

En el primer periodo (1993-1997) se aplicaron las medidas de enfrentamiento a la crisis económica y que tuvieron efectos positivos sobre el crecimiento económico. La situación especial de la economía en ese momento de crisis estructural e inserción internacional obligó a los dirigentes cubanos a instrumentar una estrategia que llevaba asociada la búsqueda de nuevos socios comerciales. Esa estrategia, que involucraba por primera vez en la historia republicana la ausencia de un socio comercial preferente, modificó su inserción internacional diversificando sus mercados, así como la dependencia en relación con el azúcar (García Fernández et al., 2010).

Por un lado, la dependencia en relación con el azúcar, rasgo típico de la economía de la isla por más de tres siglos, se alteró por primera vez desde inicios de 2000. Precisamente un efecto de la crisis de los noventa fue la reducción sostenida de la producción y las exportaciones del azúcar, lo que, aunado a la llamada política estatal de redimensionamiento de la industria azucarera a partir de 2002 (Nova, 2007), implicó cerrar 50\% de los ingenios azucareros, y disminuyeron sostenidamente la participación del azúcar en las exportaciones totales del país, de $57 \%$ en 1994 a $17 \%$ en 2013 (ONEI, 2015).

A diferencia del azúcar, el níquel aumentó su participación en las exportaciones totales de bienes desde mediados de los años noventa, en sustitución del azúcar como principal rubro de exportación, y llegó a un máximo en 2007, cuando comenzó a reducirse su contribución. En 1990 representaba $6.5 \%$; en $2000,34 \%$, y para 2005 llegó hasta $44 \%$ por el efecto sobre todo del incremento de los precios mundiales (BACI, 2006; ONEI, 2006). A partir de 1995 tuvo lugar un incremento sostenido de su producción, tras haber caído, en 1994, al nivel más bajo de la crisis de esa industria (27 000 toneladas), proceso que ocurría desde 1989, cuando se había alcanzado el tope de producción previo a la crisis. El crecimiento de la producción del níquel fue un resultado de las inversiones realizadas y de los cambios institucionales que reorganizaron la industria, como las asociaciones con empresas extranjeras (Marquetti, 2004). El mejor ejemplo es la presencia en esta industria de la Sherrit Corporation, empresa que opera una joint venture con una compañía cubana en el este del país. Entre 1995 y 2005 la producción de níquel se incrementó en 75\% (ONEI, 2001; ONEI, 2011). Posteriormente al último año mencionado, la producción refleja una reducción sistemática (véase cuadro 7), y su participación en las 
exportaciones totales se ha deteriorado en forma significativa por efecto de la caída tanto del precio como del volumen (7.4\% en 2013).

Los datos de la Oficina Nacional de Estadística no reflejan las exportaciones de refinado de petróleo. Cuba es un país netamente importador de crudo. A pesar de los esfuerzos realizados por el gobierno durante años, la producción nacional cubre algo más del $40 \%$ de las necesidades de consumo nacional; el resto casi en su totalidad se importa de Venezuela (MesaLago, 2015). Las exportaciones de refinado de petróleo son un fenómeno nuevo en la economía cubana, pues a diferencia de los años ochenta (véase cuadro 2), en este caso al petróleo importado se le incorpora valor adicional y se exporta como refinado de petróleo, lo que se convierte en una fuente adicional de ingresos en divisas. Entre 2000 y 2013 las exportaciones de refinado de petróleo crecieron a una tasa media anual de 15.7\%, sólo superadas por los medicamentos $(29.03 \%)$ y muy superiores a la tasa de crecimiento media anual de las exportaciones totales, las cuales apenas crecieron en ese mismo lapso. En términos de valores absolutos, ambos rubros representaron más de 500000000 de dólares anuales desde 2011 hasta 2013 (véase cuadro 8, anexo 1).

En cambio, se aprecia que el refinado de petróleo, los medicamentos y el laminado de tabaco han aumentado su contribuciones totales (más de 30\% en 2013 frente a 17.6\% en el 2000) (véase cuadro 7), a cuenta del azúcar y el níquel, los cuales entre ambos representaban apenas $21 \%$ del total con tendencia a disminuir aún más.

Sin embargo, la nueva situación política de Venezuela y sus condiciones económicas desde 2014 -acentuadas en 2015 por la caída del precio del petróleo, la contracción económica (de 4 y 7\% en los dos años respectivamente), la hiperinflación (la CEPAL presume que fue de tres dígitos en 2015 y $68.5 \%$ en 2014), la fuga de capitales, la deuda externa elevada y la disminución de las reservas internacionales $(31.8 \%$ del total, la segunda más elevada de la región) (CEPAL, 2016)-, seguramente influirán en el corto plazo en las exportaciones de petróleo venezolano a Cuba. De hecho estas exportaciones se redujeron absolutamente desde 2013 (Mesa-Lago, 2015), lo que afectó las exportaciones cubanas de refinado de petróleo y por consiguiente también los ingresos por exportaciones de este producto, muy importante en la balanza comercial de la isla y garante de su frágil estabilidad económica.

A pesar de que las exportaciones de medicamentos (productos de la industria médico- farmacéutica) se han incrementado sensiblemente, desde fines de los años ochenta, las exportaciones de bienes de esta industria representan aún una proporción pequeña del total de bienes exportados (véase cuadro 7). Hasta la actualidad, su principal mercado en estos productos es Venezuela, lo que refleja una relación de principal socio comer- 


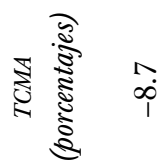

ְִ

คి

10

๙

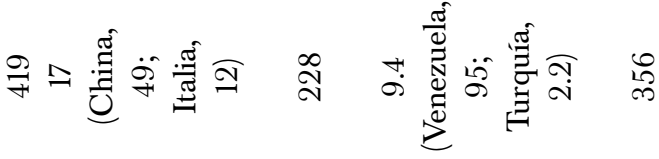

ำ

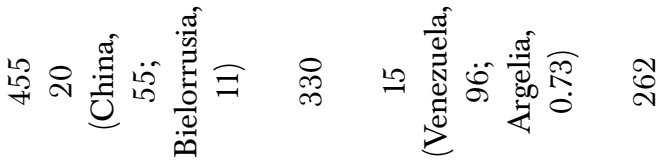

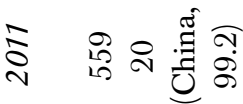

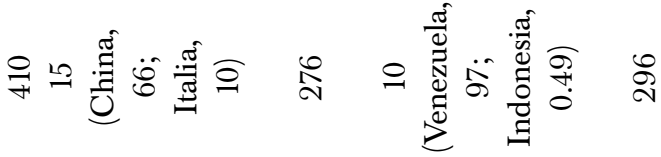

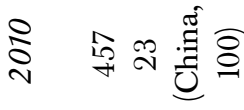

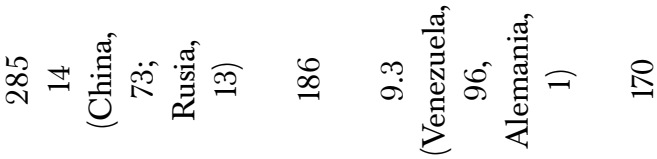

จ

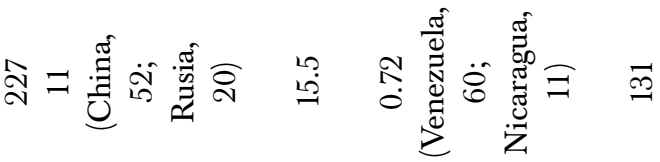

吃

受灵

독

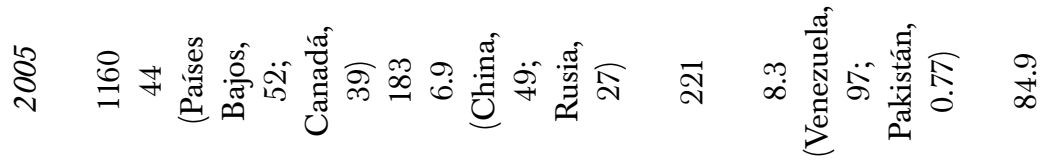

崚

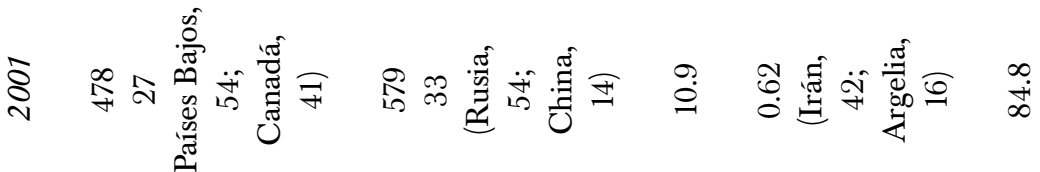

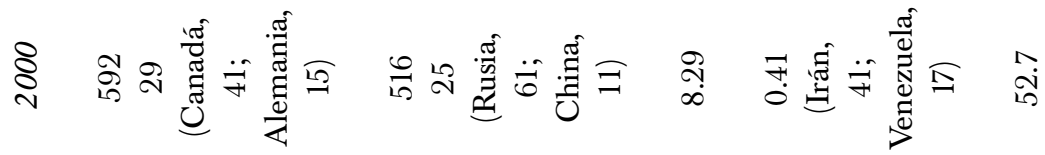

点

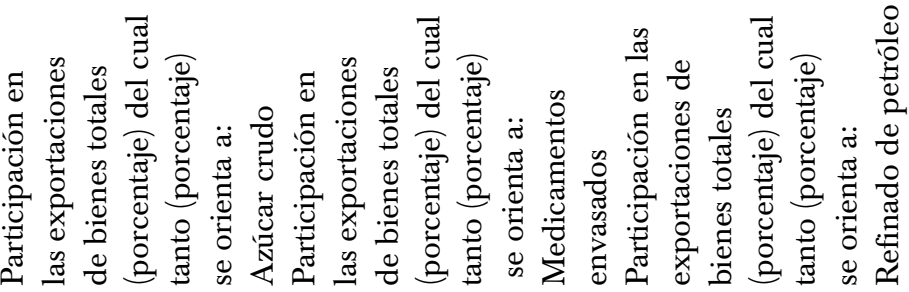


\%

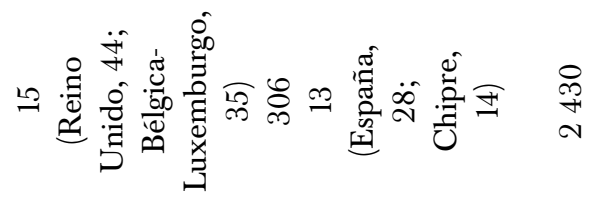

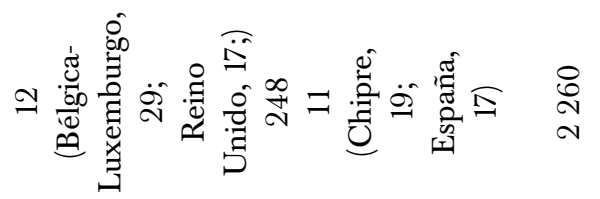

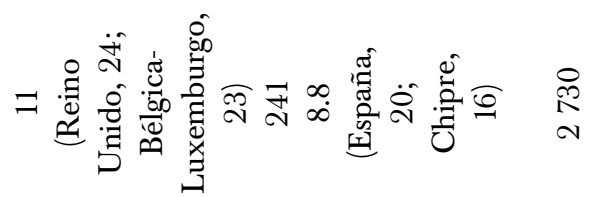

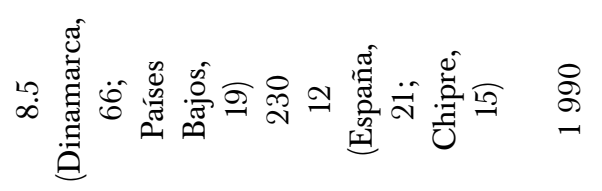

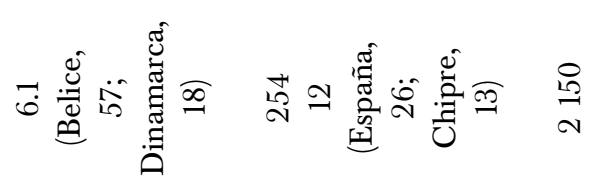

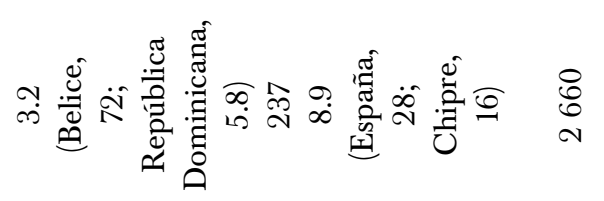

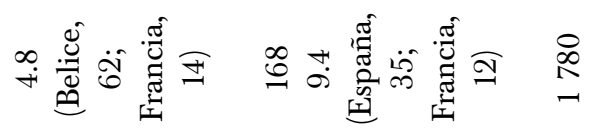

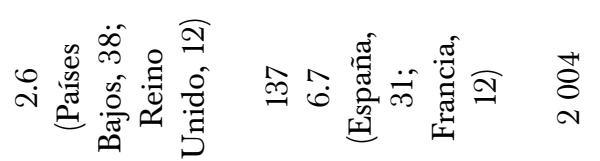

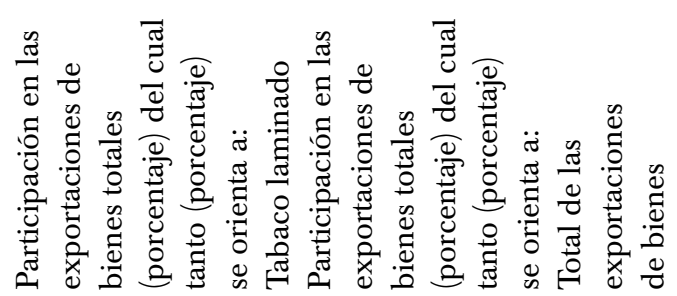


cial y, a su vez, coloca a Cuba en una situación de riesgo elevado por la incertidumbre que produce depender de ese mercado dadas sus características de inestabilidad política y económica.

A partir del año 2000, en particular con la expansión de las relaciones económicas y políticas con Venezuela, las exportaciones de servicios crecieron exponencialmente, y así compensaron el déficit permanente de la balanza de bienes. En 2004 la balanza de bienes y servicios logró por primera vez un saldo positivo, que se mantuvo hasta 2013 (con la excepción del año 2008) gracias a la aportación de la expansión de la exportación de servicios en el comercio exterior cubano. Según las cifras estimadas por el Sistema Económico Latinoamericano (en adelante SELA, 2013), la exportación de servicios cubanos pasó de 8566400000 dólares en 2008 a 11704 millones en 2012, para un crecimiento de $36.6 \%$ en tres años.

El impulso a la exportación de servicios fue promovido desde mediados de la década de 1980 -el turismo sobre todo-, como una fuente de ingreso complementaria ante la escasez de recursos externos y la necesidad de materias primas y bienes manufacturados importados. A partir de los años noventa los servicios se conviertieron en la fuente fundamental de ingresos externos conjuntamente con las remesas familiares, y ya no como ingresos complementarios, lo que ha permitido reducir la vulnerabilidad externa de la economía del país (Martín y Torres, 2007). El resto de los servicios profesionales también desempeña en la actualidad el mismo papel: generar divisas (o bienes como petróleo), que permitan mantener una capacidad de compra externa para aliviar el déficit de la balanza de bienes.

Sin embargo, el incremento de la exportación de servicios y la reducción que ha tenido lugar desde principios de los años noventa de la industria en el PIB, como expresión de un proceso de desindustrialización al estilo de los procesos neoliberales de los años ochenta en la mayoría de los países de América Latina, puede tener efectos negativos de mediano y largo plazos que probablemente no se han tomado en cuenta lo suficiente en la instauración de las políticas económicas en Cuba. Desde la perspectiva de Kaldor (1976), el éxito de los países avanzados se debe al desarrollo alcanzado en la industria. Las leyes económicas de Kaldor se refieren a los efectos positivos que genera la expansión del producto manufacturero en el conjunto de la economía al inducir el crecimiento del resto de los sectores y elevar la productividad en todas las actividades económicas. Por consiguiente, desistir de la industrialización -forzadamente o no, debido a las circunstancias de crisis de los años noventa- significa renunciar a los efectos positivos en términos de generación de empleo, transformación económica y tecnológica y generación de conocimiento especializado, cuya trascendencia es decisiva para el crecimiento económico, en tanto 
que el sector servicios no tiene la misma capacidad transformadora para el resto de la economía del país.

La exportación de servicios es la base de la especialización del país en su comercio con los países de la AlBA. Comprende la exportación de servicios profesionales (médicos y formativos-educativos) cuyo destino son principalmente Venezuela, el resto de los países de la AlBa y un grupo de países de África y Asia. Hay otro conjunto de servicios, los llamados servicios intensivos en conocimiento, que desde fines de los años noventa han evolucionado a tasas muy elevadas en la mayoría de la economías del mundo desarrollado y en las economías emergentes; sin embargo no tienen la misma presencia en Cuba ni participan tampoco del perfil de especialización de la economía del país (Martín y Torres, 2007).

Desde el punto de vista económico, la exportación de servicios ha permitido modificar el perfil de especialización internacional de la economía cubana al diversificar las fuentes de financiamiento externo y lograr un superávit en la balanza de bienes y servicios del país. No obstante, este modelo de integración tiene una debilidad básica, una alta concentración en pocos socios y depende en gran medida de las relaciones políticas que el gobierno cubano ha establecido con otros gobiernos afines ideológicamente; por consiguiente su sostenibilidad en el largo plazo no está garantizada (SELA, 2013). Como ocurre en el caso de Venezuela, Brasil y otros gobiernos con los cuales se mantienen estrechas relaciones comerciales y políticas, un cambio de gobierno puede alterar las condiciones que existían previamente en las fuerzas políticas y provocar que se prescinda de los servicios importados. Aunque Cuba ha ampliado su cartera de socios, la posible desaparición de algunos de sus socios actuales, como es el caso de Venezuela, puede tener efectos negativos para su economía, con este país en particular, donde los servicios profesionales que se exportan sirven para tener acceso a la compra de petróleo a precios más bajos que los del mercado mundial y a unas condiciones de pagos preferenciales.

La exportación tanto de servicios como de productos de la industria farmacéutica cubana son las áreas más dinámicas del sector externo. Conjuntamente con el turismo, han sido destino de importantes inversiones de recursos materiales y humanos desde fines de los años ochenta. Ambas, y sobre todo los servicios, se han expandido a tasas muy elevadas, lo que ha hecho posible un superávit externo en las cuentas públicas desde 2004.

\section{CONCLUSiOnes}

En este trabajo se ha analizado la evolución del sector exportador de bienes y servicios de la economía cubana desde 1959. Cuba tiene una eco- 
nomía abierta, por lo que el intercambio externo desempeña un papel fundamental para el éxito de las políticas económicas y el bienestar social esperado. Antes de 1959 el país estuvo inserto en un contexto de relaciones económicas, tecnológicas y financieras que cambiaron drásticamente por el curso que tomó la revolución cubana en 1959. Sin embargo, a diferencia de lo previsto al inicio, debido a la radicalidad de los cambios económicos, institucionales y políticos de los años sesenta no cambió el perfil de especialización externa de la economía cubana, pues el país continuó exportando materias primas, en particular azúcar y otros productos básicos a los países de Europa del Este y la Unión Soviética. En el llamado periodo especial ocurre un cambio deliberado en el sector exterior, aunque algunos rasgos se mantienen inalterables. La dependencia de la economía cubana de las exportaciones de azúcar, que marcaron varios siglos de historia económica, desapareció en el curso de un corto periodo (2006) y dejó de ser uno de los principales actores del mercado azucarero mundial. Se ha ido configurando un perfil internacional de la economía cubana donde tiene mayor presencia la exportación de servicios profesionales, en tanto las exportaciones de bienes pierden anualmente importancia significativa. Se ha considerado que esta nueva situación es producto de la intención del gobierno de establecer un perfil de especialización externo que sea congruente con las necesidades de sus socios actuales, por ejemplo en el marco del ALBA. Sin embargo, se ha señalado que la especialización alcanzada en el contexto de las nuevas relaciones económicas internacionales desde 1990 a la fecha contiene vulnerabilidades que pueden afectar el desempeño de la economía cubana en el corto y mediano plazos, debido a la dependencia generada en relación con sus nuevos socios, con los cuales se ha establecido una relación económica sustentada en afinidades políticas que difícilmente se podrían reorientar hacia nuevos socios comerciales.

\section{ANEXo 1. CÁlCUlO DEL ÍNDICE DE GALTUNG}

El índice Galtung se ha considerado un indicador de dependencia, el cual describe la composición del intercambio. Sus valores van de -1 a +1 . Cuando el índice se mueve hacia -1 , tanto mayor es la exportación de bienes primarios y la importación de bienes manufactureros.

La fórmula propuesta por Galtung (1971, p. 116) en sustitución de la que había propuesto K. Hongro (sugerida por el mismo Galtung) es:

$$
\mathrm{G}=\frac{(\mathrm{a}+\mathrm{d})-(\mathrm{b}+\mathrm{c})}{(\mathrm{a}+\mathrm{d})+(\mathrm{b}+\mathrm{c})}
$$


Donde $\mathrm{a}=$ valor de las materia primas importadas; $\mathrm{b}=$ valor de las materias primas exportadas; $\mathrm{c}=$ valor de los bienes manufactureros importados; $\mathrm{d}=$ valor de los bienes manufactureros exportados.

El índice Galtung ha mejorado (véase cuadro 8), al pasar de -0.53 en 1970 a -0.37 en 1989. Según LeoGrande (1979, p. 11), el índice hubiese sido mayor en valor absoluto (más cercano a -1) si Cuba no hubiera importado muchos bienes primarios. El incremento de las importaciones de petróleo, factor que sin duda acentúa el grado de dependencia de la economía cubana, ha reducido este indicador en valor absoluto. Esto significa que el valor del índice Galtung debe evaluarse en conjunto con el análisis del grado de diversificación de las exportaciones cubanas.

A diferencia de los años 1958-1989, cuando el índice Galtung mejoró, en el periodo posterior hasta 2013 ocurrió lo contrario, al pasar de -0.25 en 1994 a -0.41 en 2011, aunque en algunos años se elevó más allá a -0.46 y llegó casi al mismo nivel de 1958: -0.52. Esto probablemente es resultado del incremento que se produjo en las importaciones de bienes manufacturados y en las exportaciones de materias primas, y que acentuó el grado de dependencia de la economía cubana en relación con la exportación de commodities. Sin embargo, el análisis de todo su comercio exterior en su conjunto refleja que, si bien ese estado dominó la economía del país durante los años noventa e inicios de 2000, con posterioridad la situación ha cambiado.

\section{LISTA DE REFERENCIAS}

Banco Nacional de CUba (BNC) (1986). Informe Económico. La Habana: Autor. Banco NaCional de Cuba (BNC) (1994). Informe Económico. La Habana: Autor. Comisión Económica para América latina y el Caribe (CePAl) (2016). Balance preliminar de las economías de América Latina y el Caribe 2015. Santiago de Chile: Autor. Recuperado de http://repositorio.cepal.org/bitstream/handle/11362/39558/ S1501387_es.pdf?sequence $=98$

Comité Estatal de Estadística (1985). Anuario Estadístico de Cuba. La Habana: Autor. Comité Estatal de Estadística (1989). Anuario Estadístico de Cuba. La Habana: Autor. BACI, UN COMTRADE (2000-2013). United Nations Statistical Division (COMTRADE). Recuperado de http://atlas.media.mit.edu/es/visualize/tree_map/hs92/export/cub/ all/show/2005/

Galtung, J. (1971). A structural theory of imperialism. Journal of Peace Research, 8(2), 81-117.

García Fernández, F., López, J. L. y Sovilla, B. (2010). La crisis estructural de la economía cubana en los 90 y 2000: una interpretación heterodoxa. Revista Iberoamericana, 32(1), 15-32. 
CUADRO 8. ÍNDICE GALTUNG, 1959-2013

\begin{tabular}{llll}
\hline Año & Valor absoluto & Año & Valor absoluto \\
1958 & & & \\
1970 & -0.53 & 1995 & -0.54 \\
1975 & -0.6 & 1996 & -0.43 \\
1980 & -0.54 & 1997 & -0.47 \\
1981 & -0.52 & 1998 & -0.54 \\
1982 & -0.5 & 1999 & -0.54 \\
1983 & -0.47 & 2000 & -0.57 \\
1984 & -0.46 & 2005 & -0.47 \\
1985 & -0.42 & 2007 & -0.49 \\
1986 & -0.4 & 2008 & -0.47 \\
1987 & -0.4 & 2009 & -0.39 \\
1988 & -0.38 & 2010 & -0.40 \\
1989 & -0.4 & 2011 & -0.41 \\
1994 & -0.37 & 2012 & -0.38 \\
& -0.25 & 2013 & -0.39 \\
\hline
\end{tabular}

Fuentes: elaboración propia con datos CEE (1989, pp. 264-297), ONE (2000), ONEI (2005, 2011, 2012, 2015).

KALDOR, N. (1976). Capitalismo y desarrollo industrial: algunas lecciones de la experiencia británica. En C. F. Díaz, S. Teitel y V. Tockman (eds.), Política económica en centro y periferia. México: Fondo de Cultura Económica.

LeoGrande, W. M. (1979). Cuban dependency: a comparison of pre-revolutionary and post-revolutionary international economic relations. Cuban Studies, 9(2), 1-28.

MAÑALICH, I. (2004). El sector agrícola cubano en la década de 1990: Un análisis de competitividad. Agroalimentaria, 10(18). Recuperado de http://erevistas.saber.ula. ve/index.php/agroalimentaria/article/view/1311

MarquetTi, H. (2004). Cuba: importancia actual del incremento de las exportaciones. En O. PÉREz, Reflexiones sobre la economía cubana (pp. 142-192). La Habana: Editorial de las Ciencias Sociales.

Martín, M. y Torres, R. (2007). La economía de servicios. La Habana: Universidad de la Habana. Recuperado de https://www.nodo50.org/cubasigloXXI/economia/ martinf_310307.pdf

Mesa-Lago, C. (1969). Availability and reliability of stastistics in socialist Cuba (Part one). Latin American Research Review, 4(1), 53-91.

Mesa-Lago, C. (1969). Availability and reliability of statistics in socialist Cuba (Part two). Latin American Research Review, 4(2), 47-81. 
Mesa-Lago, C. (1981). The economy of socialist Cuba: a two-decade appraisal. Albuquerque: University of New Mexico Press.

Mesa-Lago, C. (2012). Cuba en la era de Raúl Castro. Reformas económico-sociales y sus efectos. Madrid: Colibrí.

Mesa-Lago, C. (2013). Panorama de las reformas económico-sociales y sus efectos en Cuba. En Cuba in transition. Papers and proceedings of the Twenty-Third Annual Meeting, Association for the Study of the Cuban Economy, 23. Recuperado de http://www.ascecuba.org/publications/proceedings/volume23/pdfs/mesalago.pdf

Mesa-Lago, C. (2015). Normalización de relaciones entre EEUU y Cuba: causas, prioridades, progresos, obstáculos, efectos y peligros. Documento de trabajo, 6 . Madrid: Real Instituto Elcano. Recuperado de http://www.realinstitutoelcano. org/wps/wcm/connect/08319180484ca6fea75eff2290640bff/DT6-2015-Mesa-

Normalizacion-de-relaciones-entre-EEUU-y-Cuba-causas-prioridades-progresosobstaculos-efectos-y-peligros.pdf?MOD=AJPERES\&CACHEID $=08319180484 \mathrm{c}$ a6fea75eff2290640bff

Mesa-Lago, C. y Pérez-López, J. (1985). A study of Cuba's material product system, its conversion to the system of national accounts, and estimation of gross domestic product per capita and growth rates. The World Bank, Working Papers, 770.

Nova, A. (2007). Redimensionamiento de la agroindustria azucarera cubana y el mercado internacional del azúcar. En O. E. PÉREz, Reflexiones sobre la economía cubana. La Habana: Ciencias Sociales.

NovA, A. (2015). Importancia económica y estratégica de la agroindustria cubana. En M. GONZÁLEZ-CORZO, La agroindustria cañera cubana: transformaciones recientes. Nueva York: Bildner Center for Western Hemisphere Studies The Graduate Center, The City University of New York.

Oficina Nacional de Estadística (ONE) (2000, 2001). Anuario Estadístico de Cuba. La Habana: Autor.

Oficina Nacional de Estadística E Información (ONei) (2004, 2005, 2006, 2011, 2012, 2015). Anuarios Estadísticos de Cuba. La Habana: Autor.

PÉrez-LóPez, J. (1991a). The economics of Cuban sugar. Pittsburgh: University of Pittsburgh Press.

PÉrez-LóPEZ, J. F. (1991b). Bringing the Cuban economy into focus: conceptual and empirical challenges. Latin American Research Review, 26(3), 7-53.

QuiÑones, N. y RubierA, F. (2008). Evolución del déficit por cuenta corriente de la economía cubana. ¿Han resuelto los cambios de orientación comercial y el crecimiento de servicios los desequilibrios externos de Cuba? Tribuna de Economía, 845, 185-207.

Rodríguez, J. L. (1988). Crítica a nuestros críticos. La Habana: Editorial de Ciencias Sociales.

Rodríguez, J. L. (1992). Conferencia sobre las relaciones económicas entre Cuba y la URSS. Curso de posgrado en el Centro de Estudios de las Américas (CEA), La Habana. 
Sistema Económico Latinoamericano (SELA) (2013). Análisis y recomendaciones para fomentar el comercio entre la República de Cuba y los países de América Latina y el Caribe. Caracas: Relaciones Interregionales. Recuperado de http://www.sela.org/ attach/258/EDOCS/SRed/2013/10/T023600005452-0-Di_12_-_Analisis_y_recomendaciones_para_fomentar_el_comercio_entre_CUBA_y_ALC_Rev_3_ SEP18.pdf

Zimbalist, A. (1990). Cuba's economic diversification: progress and shortcomings. En S. HALEBSKY y J. M. KIRK (eds.). Transformation and struggle: Cuba faces the 1990's (pp 131-142). Nueva York: Praeger.

Zimbalist, A. y BRundenius, C. (1989). The Cuban economy. Measurement and analysis of a socialist performance. Baltimore: Johns Hopkins University Press. 